УДК 616-008.63

DOI: $10.26435 /$ UC.V0I1(34).439

\author{
А.Н. Колесников ${ }^{1}$, М.Ю. Осканова², В.Д. Слепушкин ${ }^{3}$, Л.Г. Кварацхелия ${ }^{1}$ \\ ${ }^{1} Г 00$ ВПО «Донецкий национальный медицинский университет имени М. Горького», Донецк \\ 2Республиканская клиническая больница Республики Ингушетия, Назрань, Россия \\ ${ }^{3}$ ФББОУ ВПО «Северо-Осетинская государственная медицинская академия» Минздрава России, Владикавказ, Россия
}

\title{
ОЦЕНКА ИСХОДНОГО СТАТУСА ПАЦИЕНТОВ С ПОЛИТРАВМОЙ КАК ОСНОВНОЙ КОМПОНЕНТ СТРАТЕГИИ АНАЛГОСЕДАЦИИ ПАЦИЕНТОВ В ОИТ
}

\begin{abstract}
Известно, что хирургическое вмешательство вызывает воспалительную реакцию [1], величина которой зависит от типа операции и степени повреждения ткани [2]. Эта послеоперационная воспалительная реакция сопровождается депрессией клеточного иммунитета, что в свою очередь предрасполагает к послеоперационным инфекциям и сепсису у пациентов [3]. Кроме того, иммуносупрессия также вызывается различными препаратами, применяемыми во время анестезии $[4,5]$. Влияние анестетиков на воспалительные цитокиновые профили ранее было определено в основном у хирургических пациентов или пациентов с критическими заболеваниями, когда иммунологический статус неизбежно был изменен из-за хирургического вмешательства, совместного лечения и / или основного заболевания [6-8]. Всё это необходимо учитывать при выборе препаратов для анестезии и седации, поскольку все они сложно взаимодействуют друг с другом и способны вызывать не только положительный, но и отрицательный эффект.
\end{abstract}

Еще одной проблемой, выявленной нами, является то, что оценка влияния препаратов в ОИТ начинается как бы «с чистого листа», без учета уже имеющихся изменений в соматическом, иммунном и гормональном состояниях, связанных как с самим фактом травмы, так и с фактом оперативного вмешательства и уже имеющимся влиянием препаратов для общей анестезии на пациентов с политравмой.

Дополнительной проблемой является еще и обезболивание. Однако эта проблема далека от разрешения по причине непредсказуемого взаимодействия анальгетических и седативных препаратов, особенно в условиях динамически меняющегося критического состояния. В результате термин «анальгоседация» на какомто этапе утратил свое первоначальное значение как вариант мультимодальной анестезии. И вра- чи, и, к сожалению, многие исследователи оценивают либо седацию (на фоне базовой аналгезии), либо только анальгетики (без учета компонентов седации). Однако, по нашему мнению, адекватная аналгоседация пациентов в ОИТ не ограничивается только лишь аналгезией или седацией, а включает в себя целый ряд мероприятий, направленных на обеспечение защиты пациента от стресса, восстановление функций органов с последующим выздоровлением.

\section{ЦЕЛЬИССЛЕДОВАНИЯ}

Определить исходный статус пациентов с политравмой при поступлении в ОИТ для разработки превентивной стратегии аналгоседации, что выполняется впервые.

\section{МАТЕРИАЛ И МЕТОДЫ}

В исследовании участвовали 78 пациентов с политравмой, находящихся в ОИТ, из которых 49 составили мужчины (62,8\%), и 29 (37,2\%) женщины. Средний возраст $(\mathrm{M} \pm \mathrm{m})$ всех пациентов - 52,2 $\pm 3,5$ года.

Сбор анамнеза и жалоб пациентов включал в себя оценку по шкалам SOFA, GCS; учитывая необходимость оценки неврологических дефицитов, использовалась унифицированная для взрослых и детей модифицированная шкала Рэнкин (MRS - The Modified Rankin Scale), для диагностики степени когнитивных нарушений - шкала «Краткая шкала оценки психического статуса - MMSE (Mini Mental Score Examination)».

Интерпретация результатов MMSE: результат теста получали, суммируя результаты по каждому из пунктов. Максимально в этом тесте можно

(c) А.Н. Колесников, М.Ю. Осканова, В.Д. Слепушкин, Л.Г. Кварацхелия, 2020

(c) Университетская Клиника, 2020 
набрать 30 баллов, что соответствует наиболее высоким когнитивным способностям. За относительную норму принимали оценку по MMSE более 26 баллов. Оценку 21-25 баллов принимали за умеренные когнитивные нарушения, а менее 20 - за тяжелые когнитивные нарушения.

Модифицированная шкала Рэнкина (The Modified Rankin Scale, MRS) позволяет объективизировать динамику неврологических симптомов и функциональных нарушений. Уникальность данной шкалы состоит в возможности использования ее как у взрослых, так и у детей, а также в возможности оценивать неврологические дефициты не только после инсульта, но и после любой церебральной недостаточности.

Для описания основных функциональных систем использовали шкалу SOFA как наиболее адаптированную к возможностям клинических лабораторий тех учреждений, где проводилось исследование.

Шкала комы Глазго (ШКГ, The Glasgow Coma Scale, GCS) использовалась в исследовании не только в составе оценки SOFA, но и как основная для нейрохирургической группы пациентов.

Проводились исследования для оценки степени нейродегенерации и нейрорепарации (нейроспецифические белки). Белок S-100 (глиальный) набор предназначен для количественного определения опухолевого маркера белка S-100B (S-100A1B + S-100BB) в образцах сыворотки иммуноферментным методом для оценки степени нейродегенерации. Данный метод Fujirebio S100 EIA - основывается на применении двух различных видов моноклональных антител: биотинилированных анти-S-100B, моноклональных антител S-23 и моноклональных анти-S-100B антител MAb S-53, конъюгированных с пероксидазой хрена, специфически распознающих разные эпитопы молекулы S-100B.

Цилиарный нейротрофический фактор (нейроны и глия) (CNTF) изучаляс как как фактор нейрорегенерации. Набор Human CNTF Quantikine ELISA Kit предназначен для количественного определения человеческого цилиарного (глиального) нейротрофического фактора (CNTF) в образцах супернатантов клеточных культур, сыворотки, плазмы крови методом иммуноферментного анализа. Диапазон измерения - 8-2000 пг/мл. Аналитическая чувствительность -8 пг/мл.

Статистическая обработка данных. Полученные данные подвергали статистической обработке, используя программу STATISTICA 6.0. Оценку количественных показателей на нормальность распределения проводили при помощи критерия согласия Колмогорова-Смирнова (с поправкой Лиллиефорса). Количественные показатели представлены в виде $\mathrm{M} \pm \mathrm{sd}$, где $\mathrm{M}$ среднее арифметическое значение, sd - стандартное отклонение, также определяли медиану (Me), минимум и максимум значения.

Так как закон распределения исследуемых количественных показателей отличался от нормального, статистическую значимость различий проверяли при помощи критерия КраскаллаУоллиса (в случае множественных независимых выборок). В случае зависимых совокупностей использовали W-критерий Уилкоксона. Для оценки различия между группами был применен метод расчета кратности отклонения от нормы. Для показателей, характеризующих качественные признаки, указывалось абсолютное число и относительная частота в процентах (Р\%) с ошибкой репрезентативности (m). Для проверки статистических гипотез о различиях относительных частот, долей и отношений в двух независимых выборках использовались использовался критерий $\chi^{2}$ Пирсона (с поправкой Йетса).

Во всех процедурах статистического анализа рассчитывался достигнутый уровень значимости (р), при этом критический уровень значимости принимался равным 0,05.

\section{РЕЗУЛЬТАТЫ И ОБСУЖДЕНИЕ}

В ходе исследования оценивали гемодинамику пациентов в интра- и раннем послеоперационном периоде. Интересным оказался тот факт, что гемодинамическая нестабильность

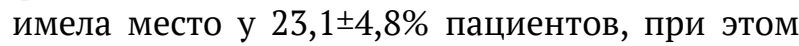
обнаружена, возможно, новая (хотя патофизиологически обоснованная) связь данных изменений с интраоперационными колебаниями BIS y $21,8 \pm 4,7 \%$ пациентов. Таким образом, было установлено, что у пациентов с травмой возможен достаточно высокий процент эпизодов гемодинамической нестабильности, и связанными с ними эпизодами неадекватности глубины анестезии (по данным BIS).

При оценке всех осложнений послеоперационного периода их можно было отнести к двум большим (часто взаимосвязанным) группам: 1) длительности пребывания на ИВЛ в послеоперационном периоде, длительности пробуждения, длительности пребывания в реанимации после операции; 2) связанными с исходными нарушениями в предоперационном и интраоперационном периодах и приводящими к развитию гнойно-септических осложнений, когнитивных расстройств.

При нормальном предоперационном состоянии пациента, нормальном интраоперационном периоде средняя длительность пребывания в ОРИТ составляет не более суток, а на ИВЛ - не более 1 часа. 
Одним из самых интересных и социально значимых параметров, изучаемых в данном исследовании, являлась когнитивная дисфункция. Так, в послеоперационном периоде выявляется когнитивная дисфункция легкой степени

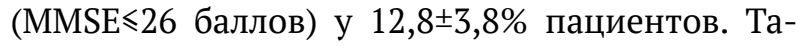
ким образом, травматический и операционный стрессы могут быть предикторами для развития когнитивных нарушений различной степени тяжести у 13\% пациентов.

Оценивая факторы нейродегенерации в виде белка S100, мы выявили, что после операции средние показатели белка S100 составляли $0,155(0,002) \mathrm{Me}=0,102(0,081-0,137)$ (значимо ниже $(\mathrm{p}<0,05)$ относительно предоперационных данных 0,194 Me=0,121). При оценке распределения показателей внутри группы было установлено, что в пределах нормы показатель на-

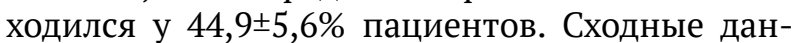
ные наблюдались и при оценке нейрорегенеративного потенциала - значение CNTF составляло 7,34(2,51) Me=4,58 (3,58-5,93) у пациентов с травмой. При оценке группового распределения зафиксировано, что в пределах нормы уровень CNTF находился у 98,9ะ1,3\% (без отличия от предоперационных данных). Следовательно, полученные данные указывают на снижение нейродегенеративных процессов в послеоперационном периоде.

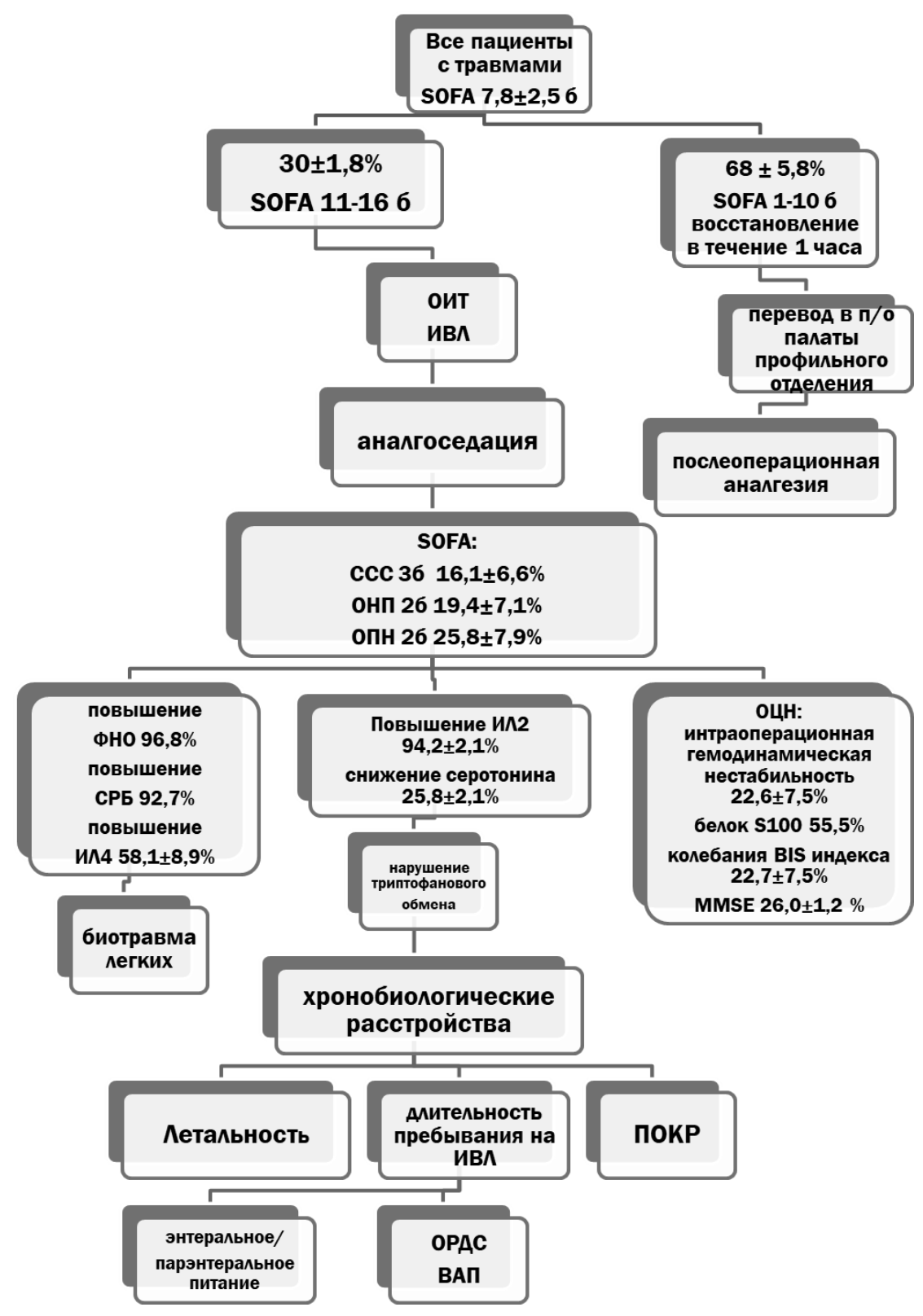

Рис. 1. Оценка исходного статуса пациентов с политравмой 
Комплексная оценка пациентов с травмой при переводе из операционной

Таблица 1. (точка отсчета начала анальгоседации)

\begin{tabular}{cccccc}
\hline \multirow{2}{*}{ Группа } & \multicolumn{5}{c}{ Шкалы исследования } \\
\cline { 2 - 6 } & $\begin{array}{c}\text { SOFA } \\
\text { (баллы) }\end{array}$ & $\begin{array}{c}\text { MRC } \\
\text { (баллы) }\end{array}$ & $\begin{array}{c}\text { GCS } \\
\text { (баллы) }\end{array}$ & $\begin{array}{c}\text { MMSE } \\
\text { (баллы) }\end{array}$ & ГCO (\%) \\
\hline \hline Травма & $7,8 \pm 2,5$ & $2,0 \pm 0,2$ & $13,0 \pm 1,0$ & $26,0 \pm 1,2$ & $32,1 \pm 5,3$ \\
\hline
\end{tabular}

Важным этапом исследования является оценка пациентов по шкале SOFA. В ходе исследования было выявлено, что средняя оценка по SOFA среди всех пациентов была 7,8 $\pm 2,5$ баллов. Из них $67,9 \pm 5,3 \%$ пациентов имели оценку от 1 до 10 баллов, что соответствовало нормальным значениям. Такие пациенты восстанавливались в течение часа после операции, переводились в п/о палату и не требовали перевода в ОИТ (рис. 1.). Однако $30,8 \pm 5,2 \%$ пациентов имели оценку SOFA 11-16 баллов. Можно констатировать, что до $30 \%$ пациентов с травмами в послеоперационном периоде имели признаки полиорганной недостаточности, что и обусловило их перевод в ОИТ, проведение продленной ИВЛ и аналгоседации.

В показателях работы сердечно-сосудистой системы нарушения до 2 баллов установлены у $12,8 \pm 3,8 \%$ пациентов. В показателях работы системы коагуляции выявлено увеличение про- цента недостаточности до 3 баллов у $16,7 \pm 4,2 \%$ (больше на 5\% по сравнению с предоперационными данными), отмечено снижение на $15 \%$ пациентов без нарушений в системе коагуляции (1 балл). Также выявлены послеоперационные нарушения в работе печеночной системы с проявлениями дисфункции до 2 баллов у 19,2 $\pm 4,5 \%$ пациентов. Сходные показатели наблюдалась в работе почечной системы, когда дисфункция до 2 баллов выявлялась у $25,6 \pm 4,9 \%$ пострадавших. Комплексная оценка статуса пациентов с травмой представлена в таблице 1.

Кроме того, в данной группе проводили иммунологическую оценку исходного состояния пациентов (табл. 2.). Было выявлено повышение уровня IL2 у 75\% пациентов, тогда как у 25\% данный показатель был в пределах нормы. Поскольку IL2 повышает активность триптофан- и серотонин-деградирующего фермента - индоламин-2,3-диоксигеназы, у 25\% пациен-

Комплексная клинико-иммунологическая оценка пациентов с травмой

Таблица 2. (точка отсчета начала анальгоседации), \% пациентов

\begin{tabular}{|c|c|c|c|}
\hline Показатель & Снижение & Норма & Превышение \\
\hline Гемодинамическая нестабильность & & & $23,1 \pm 4,8$ \\
\hline САД & 15 & 55 & 30 \\
\hline Колебания BIS-индекса & & & $21,8 \pm 4,7$ \\
\hline MMSE $\leqslant 26$ баллов & & & $12,8 \pm 3,8$ \\
\hline Гипертермия (первые 24 час) & & & $32,1 \pm 5,3$ \\
\hline белок S100 & & 3 & $55,5 \%$ \\
\hline CNTF & & $98,7 \pm 1,1$ & \\
\hline SOFA & & $\begin{array}{c}0-6 \text { баллов } \\
\text { до } 9,0 \pm 3,2\end{array}$ & $\begin{array}{c}\text { 7-8 баллов }-44,9 \pm 5,6 \\
\text { 9-10 баллов }-12,8 \pm 3,8 \\
16 \text { баллов }-6,4 \pm 2,8 \\
13-14 \text { баллов }-12,8 \pm 3,8 \\
11-12 \text { баллов }-12,8 \pm 3,8\end{array}$ \\
\hline CCC & & 2 балла $12,9 \pm 6,0$ & до 3 баллов у 16,7士4,2 \\
\hline Печеночная дисфункция & & & 2 балла у $19,2 \pm 4,5$ \\
\hline Почечная дисфункция & & & 2 балла у $25,6 \pm 4,9$ \\
\hline
\end{tabular}


тов наблюдалось значительное снижение уровня серотонина в крови, требующее фармакологической коррекции.

Средние значения IL4 как представителя «противовоспалительных» цитокинов и стресслимитирующей системы был достоверно повышен в послеоперационном периоде как по отношению к предоперационным данным, так и по отношению к норме, «уравновешивая» разнонаправленные изменения в системе стрессактивирующих/провоспалительных цитокинах.

Уровень СРБ у пациентов с травмой в послеоперационном периоде демонстрировал недостоверное увеличение, по сравнению с предоперационными данными, и достоверно превышал показатели нормы.

Полученные данные свидетельствуют о сохраняющемся исходном иммунодефиците, который, вероятнее всего, связан с истощением ресурсов организма. Для борьбы с выявленным состоянием необходим комплексный подход (рис. 2.).

Так, пациенты, находящиеся на длительной ИВЛ, требуют обязательного проведения аналгоседации. Аналгоседация должна быть подобрана с учетом хронофизиологических особенностей пациентов с политравмой. Помимо этого, для предотвращения развития осложнений в виде вентилятор-ассоциированной пневмонии и биотравмы легких необходимо тщательно подбирать режимы ИВЛ. Необходимо также учи-

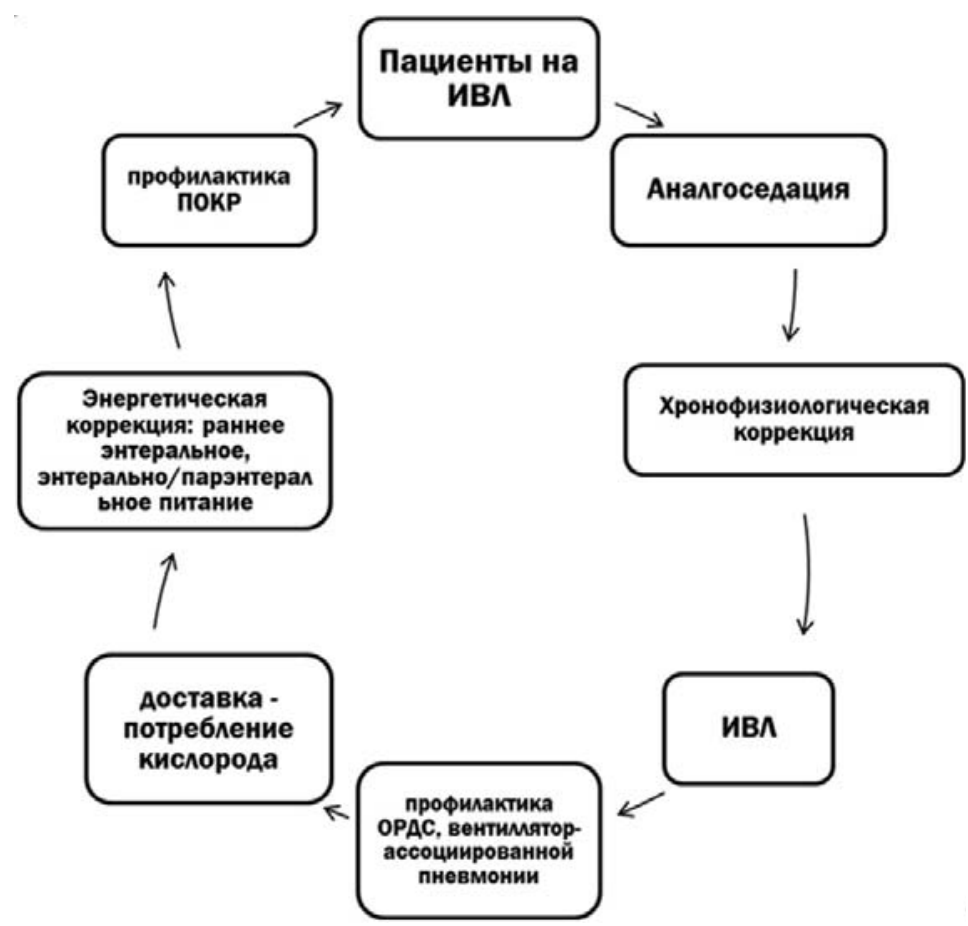

Рис. 2. Комплексный подход в стратегии седации пациентов в ОИТ тывать, что пациенты в ОИТ требуют больших затрат энергии за счет преобладания катаболических процессов в организме, поэтому следует тщательно подбирать режимы оксигенации и нутритивной поддержки пациентов. Базовым в течение первых 3-х суток (72 часа) может являться парэнтеральное питание с расчетом суточной потребности, энтеральное же питание будет рассматриваться как вспомогательное. При этом, согласно определениям, ранним является энтеральное питание в течение 48 ч. после поступления в отделение интенсивной терапии (ОИТ). Энтеральное питание следует начинать как можно скорее, потому что оно само по себе может способствовать кишечной перистальтике.

\section{З А К ЛЮЧЕНИЕ}

Проведя оценку исходного состояния пациентов с политравмой по шкале SOFA при переводе их в ОИТ, мы установили, что лишь 30\% пациентов нуждаются в длительной ИВЛ. Комплексный подход пациентов с политравмой на длительной ИВЛ включает в себя следующие знаковые моменты:

1) Первые 72 часа проведения аналгоседации необходимо признать критическими для принятия решения:

- о смене режима аналгезии;

- о смене режима седации (уменьшении доз вводимых препаратов);

- о переводе на полное энтеральное питание;

- о смене режимов ИВЛ, с переходом на режимы, поддерживающие самостоятельное дыхание.

2) Необходимо разделять режимы седации на ночные и дневные с использованием в качестве дневного седатика пропофола (в больших дозировках), в качестве ночного - тиопентала натрия или мидазолама (в меньших дозировках).

3) $75 \%$ пациентов нуждаются в «консервации сна», путем введения экзогенного мелатонина.

4) 5-е сутки терапии являются контрольными для решения вопроса об отказе от респиратора:

- у 25\% пациентов существует абсолютная необходимость в применении экзогенного серотонина. 


\section{А.Н. Колесников ${ }^{1}$ М.Ю. Осканова ${ }^{2}$ В.Д. Слепушкин ${ }^{3}$, Л.Г. Кварацхелия ${ }^{1}$}

${ }^{1}$ ГОО ВПО «Донецкий национальный медицинский университет имени М. Горького», Донецк

${ }^{2}$ Республиканская клиническая больница Республики Ингушетия, Назрань, Россия

${ }^{3}$ ФБОУ ВПО «Северо-Осетинская государственная медицинская академия» Минздрава России, Владикавказ, Россия

\section{ОЩЕНКА ИСХОДНОГО СТАТУСА ПАЦИЕНТОВ С ПОЛИТРАВМОЙ КАК ОСНОВНОЙ КОМПОНЕНТ СТРАТЕГИИ АНАЛГОСЕДАЦИИ ПАЦИЕНТОВ В ОИТ}

Известно, что хирургическое вмешательство вызывает воспалительную реакцию, величина которой зависит от типа операции и степени повреждения ткани [2]. Эта послеоперационная воспалительная реакция сопровождается депрессией клеточного иммунитета, что в свою очередь предрасполагает пациентов к послеоперационным инфекциям и сепсису. Еще одной проблемой, выявленной нами, является то, что оценка влияния препаратов в ОИТ начинается как бы «с чистого листа», без учета уже имеющихся изменений в соматическом, иммунном и гормональном состояниях, связанных как с самим фактом травмы, так и с фактом оперативного вмешательства и уже имеющимся влиянием препаратов для общей анестезии на пациентов с политравмой.

Цель исследования. Определить исходный статус пациентов с политравмой при поступлении в ОИТ для разработки превентивной стратегии аналгоседации, что выполняется впервые.

Материал и методы. В исследовании участвовали 78 пациентов с политравмой, находящихся в ОИТ, из которых 62,8\% составили мужчины и 37,2\% - женщины. Средний возраст всех пациентов - 52,2ะ3,5 года.
Сбор анамнеза и жалоб пациентов включал в себя оценку по шкалам SOFA, GCS, MRS, MMSE. Оценивались уровни белка S-100 и цилиарный нейротрофический фактор (CNTF).

Результаты и обсуждение. Оценив по шкале SOFA, мы установили, что лишь 30\% пациентов с политравмой требуют перевода в ОИТ с длительной ИВЛ и аналгоседацией. Эти пациенты пребывают в состоянии выраженного иммунодефицита и требуют комплексного подхода к лечению.

Заключение. Пациентам на длительной ИВЛ необходимо тщательно подбирать стратегию аналгоседации с учетом исходного состояния и биоритмологических особенностей. Необходимо тщательно подбирать режимы ИВЛ, следить за оксигенацией. Немаловажным этапом лечения является подбор нутритивной поддержки с наиболее ранним переходом на энтеральное питание. Заключительным этапом является индивидуальный подбор антибиотикотерапии для предотвращения осложнений ИВЛ.

Ключевые слова: аналгоседация, исходное состояние, длительная ИВЛ, нутритивная поддержка, когнитивные нарушения.

\section{A.N. Kolesnikov', M.Yu. Oskanova ${ }^{2}$, V.D. Slepushkin ${ }^{3}$, L.G. Kvaratskhelia ${ }^{1}$}

${ }^{1}$ SEI HPE «M. Gorky Donetsk National Medical University», Donetsk ${ }^{2}$ Republican Clinical Hospital of the Republic of Ingushetia, Nazran, Russia ${ }^{3}$ FSFEI HPE «Northen Ossetian State Academy of Medicine» Ministry of Health of Russia, Vladikavkaz, Russia

\section{ASSESSMENT OF THE INITIAL STATUS OF PATIENTS WITH POLYTRAUMA AS THE MAIN COMPONENT OF THE PATIENT ANALGESIA STRATEGY IN ICU}

Introduction. It is known that surgical intervention causes an inflammatory reaction, the magnitude of which depends on the type of operation and the degree of tissue damage [2]. This postoperative inflammatory reaction is accompanied by depression of cellular immunity, which, in turn, predisposes patients to postoperative infections and sepsis. Also, the problem that we identified is that the assessment of the effect of drugs in ICU starts as if "with a blank sheet", without taking into account already existing changes in somatic, immune and hormonal conditions, related both to the fact of the injury itself and to the fact of surgery and the existing effect of drugs for general anesthesia on patients with polytrauma.

Purpose of the study. To determine the initial status of patients with polytrauma upon admission to ICU to develop a preventive analgesic strategy, which is being performed for the first time.

Material and methods. The study involved 78 patients with polytrauma, located in the ICU, of which $62.8 \%$ were men and $37.2 \%$ were women. The average age of all patients was $52.2 \pm 3.5$ years. The collection of patient history and complaints included an assessment according to the scales of SOFA, GCS, MRS, MMSE. S-100 protein levels and ciliary neurotrophic factor (CNTF) were evaluated.

Results and discussions. Having performed the SOFA assessment, it was found that only $30 \%$ of patients with polytrauma require transferring to ICU with long-term mechanical ventilation and analgesia. These patients are in a state of severe immunodeficiency and require an integrated approach to treatment.

Conclusion. Patients on long-term mechanical ventilation must carefully select an analgesic strategy, taking into account the initial state and biorhythmological features. It is necessary to carefully select the ventilation mode, monitor oxygenation. An important stage of treatment is the selection of nutritional support with the earliest transition to enteral nutrition. The final step is an individual selection of antibiotic therapy to prevent ventilation complications.

Key words: analgosedation, initial state, long-term mechanical ventilation, nutritional support, cognitive impairment. 


\section{ЛИТЕРАТУРА}

1. Sherwood E.R., Toliver-Kinsky T. Mechanisms of the inflammatory response. Best Pract Res ClinAnaesthesiol. 2004; 18: 385-405.

2. Helmy S.A., Wahby M.A., El-Nawaway M. The effect of anaesthesia and surgery on plasma cytokine production. Anaesthesia. 1999; 54:733-738.

3. Angele M.K., Faist E. Clinical review: immunodepression in the surgical patient and increased susceptibility to infection. Crit Care BioMed Central. 2002; 6:298-305.

4. Kurosawa S. Anesthesia in patients with cancer disorders. Curr Opin Anaesthesiol. 2012; 25: 376-384.

5. Kurosawa S., Kato M. Anesthetics, immune cells, and immune responses. J Anesth. 2008; 22: 263-277.

6. Sanders R.D., Hussell T., Maze M. Sedation \& immunomodulation. Crit Care Clin. 2009; 25: 551-570.

7. Helmy S.A., Al-Attiyah R.J. The immunomodulatory effects of prolonged intravenous infusion of propofol versus midazolam in critically ill surgical patients. Anaesthesia. 2001; $56: 4-8$.

8. Sofra M., Fei P.C., Fabrizi L., Marcelli M.E., Claroni C., Gallucci M. et al. Immunomodulatory effects of total intravenous and balanced inhalation anesthesia in patients with bladder cancer undergoing elective radical cystectomy: preliminary results. J Exp Clin Cancer Res. 2013; 32: 6.

\section{REFERENCES}

1. Sherwood E.R., Toliver-Kinsky T. Mechanisms of the inflammatory response. Best Pract Res ClinAnaesthesiol. 2004; 18: 385-405.

2. Helmy S.A., Wahby M.A., El-Nawaway M. The effect of anaesthesia and surgery on plasma cytokine production. Anaesthesia. 1999; 54:733-738.

3. Angele M.K., Faist E. Clinical review: immunodepression in the surgical patient and increased susceptibility to infection. Crit Care BioMed Central. 2002; 6:298-305.

4. Kurosawa S. Anesthesia in patients with cancer disorders. Curr Opin Anaesthesiol. 2012; 25: 376-384.

5. Kurosawa S., Kato M. Anesthetics, immune cells, and immune responses. J Anesth. 2008; 22: 263-277.

6. Sanders R.D., Hussell T., Maze M. Sedation \& immunomodulation. Crit Care Clin. 2009; 25: 551-570.

7. Helmy S.A., Al-Attiyah R.J. The immunomodulatory effects of prolonged intravenous infusion of propofol versus midazolam in critically ill surgical patients. Anaesthesia. 2001; 56: 4-8.

8. Sofra M., Fei P.C., Fabrizi L., Marcelli M.E., Claroni C., Gallucci M. et al. Immunomodulatory effects of total intravenous and balanced inhalation anesthesia in patients with bladder cancer undergoing elective radical cystectomy: preliminary results. J Exp Clin Cancer Res. 2013; 32: 6. 